\title{
Spatial resolved temperature measurement based on absorption spectroscopy using a single tunable diode laser
}

\author{
Xilong Yu · Fei Li · Lihong Chen · Xinyu Zhang
}

Received: 4 August 2009 / Accepted: 6 August 2009 / Published online: 18 September 2009

(C) The Chinese Society of Theoretical and Applied Mechanics and Springer-Verlag GmbH 2009

\begin{abstract}
A novel method based on wavelength-multiplexed line-of-sight absorption and profile fitting for nonuniform flow field measurement is reported. A wavelength scanning combing laser temperature and current modulation WMS scheme is used to implement the wavelength-multiplexed-profile fitting method. Second harmonic (2f) signal of eight $\mathrm{H}_{2} \mathrm{O}$ transitions features near $7,170 \mathrm{~cm}^{-1}$ are measured in one period using a single tunable diode laser. Spatial resolved temperature distribution upon a $\mathrm{CH}_{4}$ /air premixed flat flame burner is obtained. The result validates the feasibility of strategy for non-uniform flow field diagnostics by means of WMS-2f TDLAS.
\end{abstract}

Keywords WMS-2f TDLAS · Profile fitting ·

Spatial resolved $\cdot$ Temperature measurement

\section{Introduction}

Near-infrared absorption spectroscopy technique is an effective method for measuring gas temperature and concentration in many combustion flows due to its advantages of high sensitivity and quick response. But because of integrated measurement along the line of sight, the application is limited to the flow field with nearly uniform properties. While in practical flow field, significant temperature gradients may exist along the optical path due to chemical reaction, strong shock wave,

The project was supported by the National Natural Science Foundation of China (10772188).

X. Yu $(\bowtie) \cdot$ F. Li $\cdot$ L. Chen $\cdot$ X. Zhang

Key Laboratory of High Temperature Gas Dynamics, Institute of Mechanics, Chinese Academy of Sciences, 100190 Beijing, China

e-mail: xlyu@imech.ac.cn boundary layer and other effects. For application of TDLAS to non-uniform flow field diagnostics, the biggest challenge is how to obtain the accuracy spatial temperature and species concentration distribution with the least lasers $[1,2]$. In the paper, a novel method, called Profile Fitting, is proposed to resolve these non-uniformities which rely on measurements of multiple absorption transitions of a single species [3]. A wavelength scanning combing laser temperature and current modulation WMS (Wavelength Modulation Spectroscopy) scheme with single fiber coupled DistributedFeedback diode laser near $1,395 \mathrm{~nm}$ is implemented to record high resolution eight absorption spectra upon slightly disturbed flat flame in a premixed $\mathrm{CH}_{4} /$ air Mckenna burner, and feasibility of diagnostic strategy is confirmed by consistence of TDLAS measurement and thermocouple results.

\section{Experimental method}

For profile fitting method, the measurement accuracy increases with the number of the well selected absorption transitions. In previous work [1], wavelength-scanning method was based on modulating the injection current which can make the laser tune over about $2 \mathrm{~cm}^{-1}$. But by changing the control temperature, it can be scanned cross more than $10 \mathrm{~cm}^{-1}$ at the manner of slow response (typical $25 \mathrm{mHz}$ repetition rate). So it is possible to cross much more transitions with a single diode laser in one scan period by means of temperature scanning strategy. This is very appropriate for the static flow measurement. Moreover, for overcoming low intensity attenuation of weak absorption transitions in directscanning multiple lines' transmission, high sensitive wavelength modulation spectroscopy (WMS) is used to acquire enough $\mathrm{S} / \mathrm{N}$ (signal to noise) ratio for the selected transitions. The advantage of this novel method, which combines 


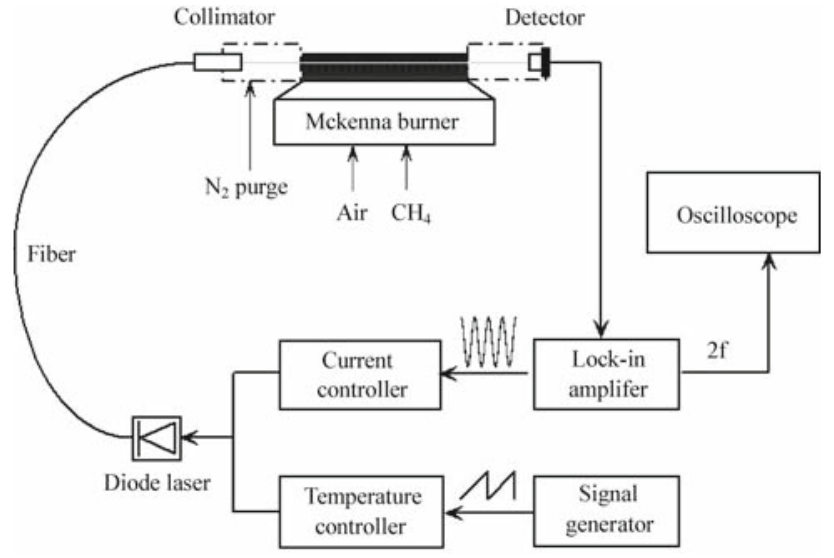

Fig. 1 Schematic of the experimental setup

wavelength-multiplexed WMS and profile fitting for spatial resolved flow field measurement using one tunable diode laser, exhibits the feature of much more transmission transitions, higher $\mathrm{S} / \mathrm{N}$ ratio for each transition, thus simple system construction and much higher accuracy for profile fitting computation. The demonstration of feasibility for the proposed sensor concept is validated in a $\mathrm{CH}_{4} /$ air premixed Mckenna flat flame burner.

Figure 1 is a schematic diagram of the wavelength-multiplexed-profile fitting system. The system is composed of a fiber-coupled DFB diode laser (NTT corporation, NLK1E5EAAA), a combined temperature and current laser controller (Thorlabs Inc., ITC502), a lock-in amplifier (SIGNAL RECOVERY, Model 7265) and a signal generator (Tektronix Inc., AFG3021). The ramp signal, with frequency $25 \mathrm{mHz}$ and amplitude 10 volt, from signal generator, is injected into temperature controller for wide range wavelength modulation. The sine signal, with frequency $500 \mathrm{~Hz}$ and amptitude 0.5 volt, generated by the lock-in amplifier, is used for current modulation and tracking reference. The combined modulation signal can control the laser wavelength modulation in $10 \mathrm{~cm}^{-1}$ range around the center wavelength and proceed $2 \times 10^{4}$ sine signal cycles in one ramp signal cycle. The transmitted laser intensity is received by a $2 \mathrm{~mm}$ diameter InGaAs detector. The reference signal and detected signal are analyzed by the lock-in amplifier, and the second harmonic signal is recorded by a memory oscilloscope (Tektronix Inc., DPO4032).

Eight water vapor transitions near $7,170 \mathrm{~cm}^{-1}$ used in this paper are listed in Table 1. Because of the close line space, the $7,170.253 \mathrm{~cm}^{-1}$ transition and $7,170.278 \mathrm{~cm}^{-1}$ transition can be treated as a combined one.

\section{Results and data analysis}

In profile fitting calculation, the shape of the nonuniform property distribution must be postulated in advance. The demonstrated experiment is carried out at a well designed premixed $\mathrm{CH}_{4}$ /air Mckenna burner. The distribution of temperature and water vapor concentration upon slightly disturbed flame is a nearly non-isosceles trapezoidal distribution along the laser beam path (as shown in Fig. 2).

The second harmonic (2f) signals of the selected transitions are shown in Fig. 3. The center heights of the $2 \mathrm{f}$ signals are used for the temperature distribution deduction. It confirms that the fitting results improve significantly when the number of unknowns (free parameters) in the postulated distribution profiles can be reduced by using physical constraints [3]. The intensity modulation coefficient $i_{0}$, the modulation depths and the optical-electrical gain coefficient $G$ were calibrated before the experiment. The total absorption length is fixed at $5 \mathrm{~cm}$. It is assumed that the shape of water vapor concentration distribution is same as that of temperature distribution. By using above physical constraints, the free parameters to be solved from the $2 \mathrm{f}$ signal are the temperature and mole fraction of the core region and the length of the two mixing layer, $T_{\mathrm{c}}, X_{\mathrm{c}}, L_{\mathrm{b} 1}, L_{\mathrm{b} 2}$.

$$
\begin{aligned}
& \min \left(f\left(T_{\mathrm{c}}, X_{\mathrm{c}}, T_{0}, X_{0}, L_{\mathrm{c}}, L_{\mathrm{b} 1}, L_{\mathrm{b} 2}, G, a, i_{0}\right)\right) \\
& =\min _{T_{\mathrm{c}}, X_{\mathrm{c}}, L_{\mathrm{b} 1}, L_{\mathrm{b} 2}} \sum_{n=1}^{7}\left(R_{2 \mathrm{f}, v_{0, n}, \text { simulation }}-R_{\left.2 \mathrm{f}, v_{0, n}, \text { experiment }\right)^{2} .}\right.
\end{aligned}
$$

Table 1 Spectroscopy parameters of the eight strong water vapor transitions near $7,170 \mathrm{~cm}^{-1}$

\begin{tabular}{lllr}
\hline $\begin{array}{l}\text { Transition } \\
\text { definition }\end{array}$ & $\begin{array}{l}\text { Frequency } \\
\left(\mathrm{cm}^{-1}\right)\end{array}$ & $\begin{array}{l}\text { Line strength at } \\
1,000 \mathrm{~K} /\left(\mathrm{cm}^{-2} \mathrm{~atm}^{-1}\right)\end{array}$ & $\begin{array}{l}\text { Low-state } \\
\text { energy }\left(\mathrm{cm}^{-1}\right)\end{array}$ \\
\hline$L_{1}$ & $7,168.437$ & 0.02218 & 173.366 \\
$L_{2}$ & 0.00273 & $2,398.382$ \\
$L_{3}$ & $7,169.826$ & 0.00105 & $1,724.705$ \\
$L_{4}$ & $7,170.253$ & 0.00418 & 206.301 \\
$L_{5}$ & $7,170.278$ & 0.00207 & $2,251.863$ \\
$L_{6}$ & $7,172.507$ & 0.01274 & $1,411.612$ \\
$L_{7}$ & $7,173.781$ & 0.00321 & $1,360.235$ \\
$L_{8}$ & $7,175.492$ & 0.00341 & $2,129.599$ \\
\hline
\end{tabular}




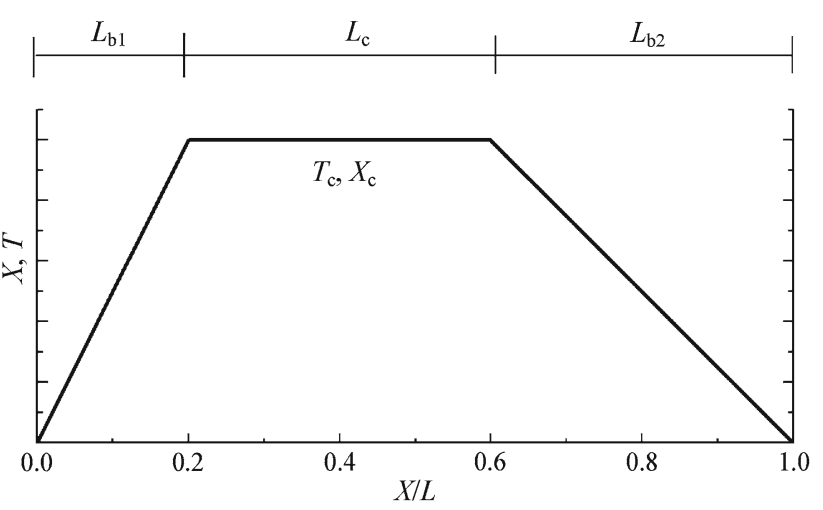

Fig. 2 Postulated temperature and water concentration distribution profiles along the optical path

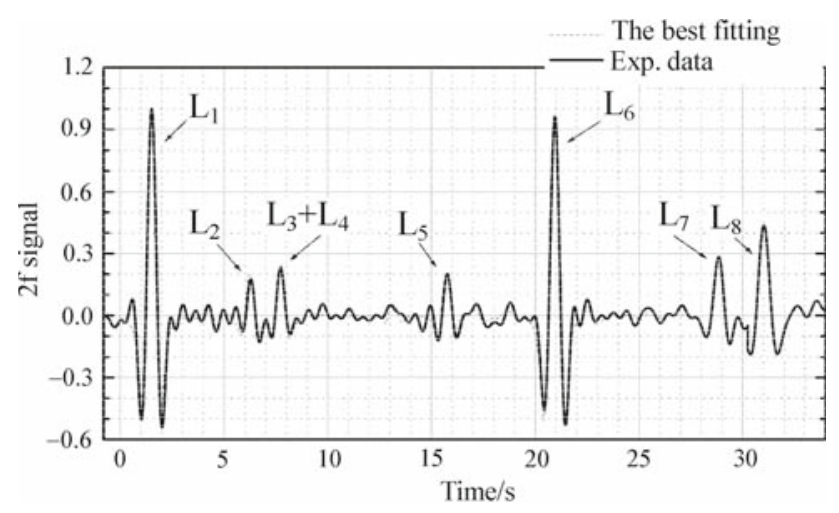

Fig. 3 Comparison of the simulated $2 \mathrm{f}$ signal of the best fitting with experimental data

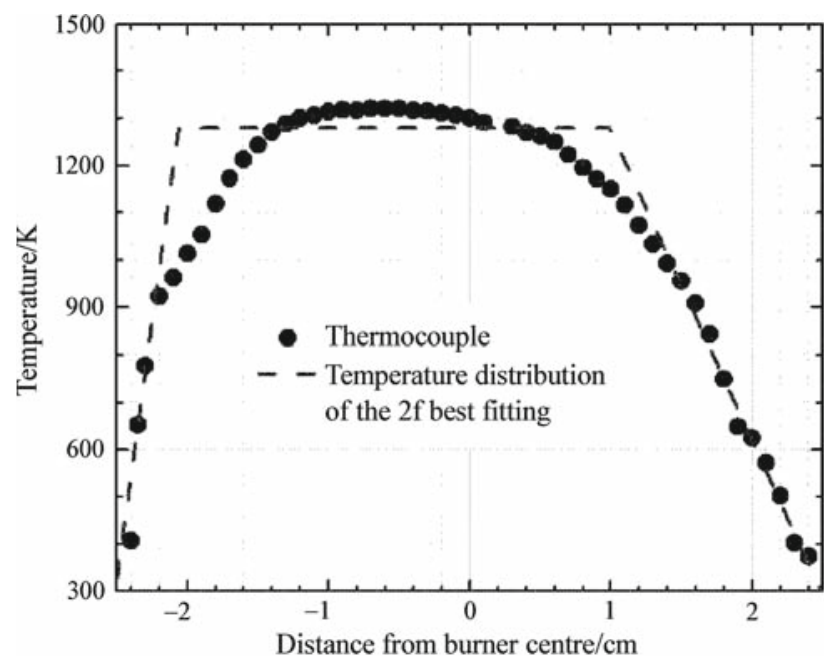

Fig. 4 Comparison of temperature distribution of the $2 \mathrm{f}$ best fitting with thermocouple measurement
Expression (1) presents the most general mathematical model for the wavelength-modulation profile-fitting method. The absorption profile and second harmonic signal $R$ of the selected lines are simulated based on HITRAN2004 [4] and the fixed physical constraints. With the best profile fitting, the parameters can be given: $T_{\mathrm{c}}=1,280 \mathrm{~K}, X_{\mathrm{c}}=20 \%, L_{\mathrm{b} 1}=$ $0.45 \mathrm{~cm}, L_{\mathrm{b} 2}=1.5 \mathrm{~cm}$.

Figure 3 presents comparison between the $2 \mathrm{f}$ signal at the best fitting state and the experiment data, which shows little difference of them. The computed temperature distribution and the thermocouple measurement results along the sight are shown in Fig. 4. The mean deviation between the profile fitting method and the thermocouple measurement is $52 \mathrm{~K}$. In the core area of flame, maximum deviation between the profile fitting method and the thermocouple measurement is $41 \mathrm{~K}(3.1 \%)$. The result illustrates the validation of WMS-2f TDLAS based profile fitting method for the spatial resolved temperature measurement.

\section{Conclusion}

The paper demonstrates the feasibility of the wavelengthmultiplexed line-of-sight absorption and the profile fitting method for the spatial resolved temperature measurement. By obtaining $2 \mathrm{f}$ signal of eight absorption features in one period using a single tunable diode laser, the potential application of the proposed method is well expand to the spatial resolved temperature measurement.

\section{References}

1. Bryner, E.B., Diskin, G.S., Goyne, C.P., McDaniel, J.C., Krauss, R.H., Slate, T.A.: Water vapor concentration measurement in high enthalpy flows using infrared absorption. Paper AIAA-2003-4580, 39th AIAA/ASME/SAE/ASEE Joint Propulsion Conference and Exhibit, 20-23 July 2003, Huntsville, Alabama

2. Cai, W.W., Ewing, D.J., Ma, L.: Application of simulated annealing for multispectral tomography. Comput. Phys. Commun. 179(8), 250-255 (2008)

3. Liu, X., Jeffries, J.B., Hanson, R.K.: Measurement of nonuniform temperature distributions using line-of-sight absorption spectroscopy. AIAA J. 45(2), 411-419 (2007)

4. Rothman, L.S., Jacquemart, D., Barbe, A., Chris Benner, D., Birk, M., Brown, L.R., Carleer, M.R., Chackerian, C., Chance, K. Jr., Coudert, L.H., Dana, V., Devi, V.M., Flaud, J.M., Gamache, R.R., Goldman, A., Hartmann, J.M., Jucks, K.W., Maki, A.G., Mandin, J.Y., Massie, S.T., et al.: The HITRAN 2004 molecular spectroscopic database. J. Quant. Spectrosc. Radiat. Transf. 96(2), 139-204 (2005) 